\title{
Randomized Controlled Trial of Oral Antifungal Treatment for Severe Asthma with Fungal Sensitization The Fungal Asthma Sensitization Trial (FAST) Study
}

\author{
David W. Denning 1,2, B. Ronan O'Driscoll3, Georgina Powell1,2, Fiona Chew ${ }^{1,2}$, Graham T. Atherton ${ }^{1,2}$, \\ Aashish Vyas ${ }^{4}$, John Miles, Julie Morris ${ }^{6}$, and Robert M. Niven ${ }^{1,2}$ \\ ${ }^{1}$ School of Translational Medicine, University of Manchester, and ${ }^{2}$ North West Lung Centre, University Hospital of South Manchester, Manchester; \\ ${ }^{3}$ Respiratory Medicine, Salford Royal Foundation NHS Trust, Salford; ${ }^{4}$ Preston Royal Hospital, Preston, Lancashire; ${ }^{5}$ North Manchester General \\ Hospital, Manchester; and ${ }^{6}$ Department of Medical Statistics, University Hospital of South Manchester, Manchester, United Kingdom
}

\begin{abstract}
Rationale: Some patients with severe asthma are immunologically sensitized to one or more fungi, a clinical entity categorized as severe asthma with fungal sensitization (SAFS). It is not known whether SAFS responds to antifungal therapy.

Objectives: To evaluate the response of SAFS to oral itraconazole.

Methods: Patients with severe asthma sensitized to at least one of seven fungi by skin prick or specific IgE testing were recruited. All had total IgE less than $1,000 \mathrm{IU} / \mathrm{ml}$ and negative Aspergillus precipitins. They were treated with oral itraconazole (200 mg twice daily) or placebo for 32 weeks, with follow-up for 16 weeks.

Measurements and Main Results: The primary end point was change in the Asthma Quality of Life Questionnaire (AQLQ) score, with rhinitis score, total $\lg \mathrm{E}$, and respiratory function as secondary end points. Fifty-eight patients were enrolled, of whom $41 \%$ had been hospitalized in the previous year. Baseline mean AQLQ score was 4.13 (range, $1-7)$. At 32 weeks, the improvement (95\% confidence interval) in AQLQ score was $+0.85(0.28,1.41)$ in the antifungal group, compared with a $-0.01(-0.43,0.42)$ change in the placebo group $(P=$ $0.014)$. Rhinitis score improved $(-0.43)$ in the antifungal, and deteriorated $(+0.17)$ in the placebo group $(P=0.013)$. Morning peak flow improved $(20.8 \mathrm{~L} /$ minute, $P=0.028)$ in the antifungal group. Total serum IgE decreased in the antifungal group $(-51 \mathrm{IU} / \mathrm{ml})$ but increased in placebo group $(+30 \mathrm{IU} / \mathrm{ml})(P=0.001)$. No severe adverse events were observed, but seven patients developed adverse events requiring discontinuation, five in the antifungal group. Conclusions: SAFS responds to oral antifungal therapy as judged by large improvements in quality of life in about $60 \%$ of patients.

Clinical trial registered with www.controlled-trials.com (ISRCTN61552714).
\end{abstract}

Keywords: Aspergillus; Cladosporium; Candida; Alternaria; Penicillium

Fungi interact directly with the human host in numerous ways. There is daily exposure to conidia, spores, hyphae, fragments of hyphae, cell wall constituents such as glucan, and volatile organic compounds, usually with little or no consequence. However, numerous studies over the last 100 years have linked worsening asthma with fungal exposure (1). Whether fungal exposure is directly relevant to the pathogenesis of asthma and its severity, or is simply a paraphenomenon, has been difficult to ascertain. A direct demonstration of efficacy of antifungal therapy for asthma

(Received in original form May 15, 2008; accepted in final form October 20, 2008) Supported by the Molton Trust, a U.K. charity, with a contribution from the Fungal Research Trust, another U.K. charity. Neither charity played any part in the data collection or analysis or contributed to writing the article.

Correspondence and requests for reprints should be addressed to D. W. Denning, F.R.C.P., Education \& Research Centre, University Hospital of South Manchester, Southmoor Road, Manchester M23 9LT, UK. E-mail: ddenning@manchester.ac.uk This article has an online supplement, which is accessible from this issue's table of contents at www.atsjournals.org

Am J Respir Crit Care Med Vol 179. pp 11-18, 2009

Originally Published in Press as DOI: 10.1164/rccm.200805-7370C on October 23, 2008 Internet address: www.atsjournals.org

\section{AT A GLANCE COMMENTARY}

Scientific Knowledge on the Subject

Some patients with severe asthma are sensitized to fungi. Studies with the antifungal agent itraconazole showed benefit in allergic bronchopulmonary aspergillosis.

What This Study Adds to the Field

The results of this randomized controlled trial showed a significant quality of life benefit, a fall in $\mathrm{IgE}$, and a modest improvement in rhinitis and morning peak flow, but not $\mathrm{FEV}_{1}$, in patients with severe asthma sensitized to one of several common fungi after oral antifungal therapy.

would directly implicate fungal exposure in the pathogenesis of asthma.

A specific disease entity exists in which massively increased allergic response to the ubiquitous fungus Aspergillus fumigatus is associated with long-term colonization of the respiratory tract with this fungus, namely allergic bronchopulmonary aspergillosis (ABPA). Most of these patients have asthma, although not all. In 1991 we showed that oral itraconazole was effective in the treatment of ABPA (2). Subsequently, two randomized controlled studies extended these early observations $(3,4)$. However, most patients with severe asthma do not have ABPA and are not demonstrably colonized with fungi in the respiratory tract, yet many are sensitized to fungi such as Cladosporium species, Alternaria species, Penicillium species, Candida species, Trichophyton species, and others (1). As most of these fungi are common in air, we hypothesized that direct external exposure or low levels of colonization are sufficient to induce an allergic response and pulmonary inflammation in many patients with severe asthma (1). Support for this concept came from a small randomized study of Trichophyton sensitization with fluconazole (5) and our own empirical experiences (6). We coined the term severe asthma with fungal sensitization, or SAFS, to provide a simple clinical label for a particular phenotype of severe asthma, with possible therapeutic implications (1). We report here the results of this randomized, blinded study, which was designed as a proof-of-concept study; some of these results were presented in abstract form in late 2007 (7).

\section{METHODS}

At four U.K. hospitals, patients with severe asthma (British Thoracic Society level 4 or 5 [8]) who were taking high-dose inhaled corticosteroids (beclometasone equivalent dose, $1000 \mu \mathrm{g} / \mathrm{d}$ or more; fluticasone, $500 \mu \mathrm{g} / \mathrm{d}$ or more), continuous oral corticosteroids (at least $5 \mathrm{mg}$ of prednisolone or its equivalent for at least $6 \mathrm{mo}$ ), or at least four or 
six courses of oral/intravenous corticosteroids over the previous 12 or 24 months, were screened for fungal sensitization by skin prick testing and RAST to the following fungi: Aspergillus fumigatus, Cladosporium herbarum, Penicillium chrysogenum (notatum), Candida albicans, Trichophyton mentagrophytes, Alternaria alternata, and Botrytis cinerea. Skin test reagents were obtained from Allergopharma (Reinbeck, Germany) and RAST tests from Phadia (Uppsala, Sweden). Those who were positive by skin prick (wheal at least $3 \mathrm{~mm}$ in diameter) or RAST (at least $0.4 \mathrm{IU} / \mathrm{ml}$ ) testing were eligible if they had total circulating $\mathrm{IgE}$ less than $1,000 \mathrm{IU} / \mathrm{ml}$, were negative for Aspergillus precipitin (IgG), did not have recurrent bacterial lung infections that would preclude evaluation, did not have a current respiratory infection (entry deferred), had not received itraconazole in the prior 8 months, were not pregnant, had liver function tests less than three times the upper limit of normal, were not allergic to azoles, were not taking any interacting drugs that could not be stopped, were not immunosuppressed other than by corticosteroids, and did not have left ventricular dysfunction (ejection fraction less than $40 \%$ on echocardiography). The local ethics committees approved the protocol and all patients gave written informed consent. The study is registered as a clinical study as number ISRCTN61552714 at www.controlled-trials.com.

\section{Primary End Point}

The primary end point was a comparison of validated Asthma Quality of Life Questionnaire (AQLQ) score (9) between the antifungal group and placebo group at baseline and at 32 weeks. The AQLQ is scored on a scale of 1 to 7 , with 7 representing an excellent quality of life. Each patient answered the 32 questions (related to quality of life with asthma) supervised by one of the two study nurses.

\section{Treatment, Blinding, and Randomization}

Itraconazole capsules (Sporanox, $100 \mathrm{mg}$ ) were purchased from the manufacturer (Janssen Pharmaceutica, Beerse, Belgium) and overencapsulated with red capsules. Matching placebos containing cellulose (Avicel; FMC BioPolymer, Philadelphia, PA) were prepared. The finished product was prepared and packed by a licensed manufacturing unit to good manufacturing practice (GMP) standard. Each patient received two capsules, corresponding to $200 \mathrm{mg}$ of itraconazole or placebo, twice daily. A randomization schedule in 4 blocks of 16 and subsequent blocks of 8 was prepared, using a computer-generated block randomization procedure, and amounts of either placebo or itraconazole equivalent to 32 weeks of treatment were packaged in tubs in 30-day supply boxes. Enrolled patients were allocated sequential numbers by one of the four hospital pharmacies, without knowledge of the randomization sequence, which was held centrally. Plasma for itraconazole assays was collected at Weeks 2, 4, 12, and 20. Treating physicians were not informed about these results, but they were made available to the principal investigator on a monthly basis by spreadsheet to make dosage adjustments, if required.

\section{Clinical Care}

Antiasthma therapy was optimized before the start of study, as much as possible. Once enrolled in the study, a patient's therapy was maintained at the same level as much as possible. Patients were seen regularly by the study nurses and local principal investigators, and were given cell phone numbers to report any problems. All nonstudy medication was recorded at each visit. Hospitalization, exacerbations, and respiratory infections were recorded as adverse events, as were any other unusual occurrences. Exacerbations in this predominantly severe asthma population were defined on a need-for-treatment basis. Worsening of asthma/breathlessness was defined as the need for a course of oral prednisolone or, for the small number receiving maintenance prednisolone, as a need to increase the dose by $10 \mathrm{mg}$ or more. Infections were defined as a need for a course of antibiotics as deemed necessary by their primary care provider, secondary care provider if they attended the emergency room, or one of the principal investigators at study visits.

\section{Laboratory Methods}

Total and allergen-specific IgE was measured with the UniCAP system (Phadia). Itraconazole plasma concentrations were measured by bioassay, as previously described (10) in a single laboratory. Serum cortisol was measured by electrochemiluminescence immunoassay on a Roche E170 modular analyzer, as per the manufacturer's instructions (Roche, Indianapolis, IN).

\section{Data Collection}

After screening (Figure 1), baseline data were collected including asthma, medical, and sinusitis history; and physical examination including skin for fungal infection, pulmonary function tests and peak expiratory flow (PEF), total corticosteroid dose, other asthma and other treatment data, and basic biochemistry and hematology as well as total serum IgE. Participating patients were seen at 2 and 4 weeks and then monthly for the 32-week treatment period, when information about clinical status, adverse events, exacerbations, additional medication, and compliance (pill counting) was assessed. They were seen once for follow-up at 48 weeks. AQLQ score, rhinitis score (11), $\mathrm{FEV}_{1}$, and self-recorded PEF readings were assessed at Weeks 4, 16, and 32 and at 4 months of follow-up. Serum IgE and RAST tests were done at Weeks 16 and 32, and at 4 months of follow-up.

\section{Null Hypothesis and Power Calculation}

The null hypothesis was as follows: "Itraconazole has no effect as an adjunctive treatment in patients with severe asthma with a skin prick test or RAST test positive to one or more tested fungal allergens, who do not fulfill the criteria for ABPA." A statistically significant improvement in quality of life as measured by the AQLQ score at 32 weeks was the primary end point.

Sample size calculations with a two-sided $\alpha$ of 0.05 and a power of 0.8 indicated that 86 patients would be required to detect differences in AQLQ scores greater than 0.75 (assuming a common standard deviation of 0.6 ). An attrition rate of $25 \%$ was assumed and so a study size of 108 patients was planned.

\section{Study Populations}

The primary analysis population (modified intent-to-treat [MITT]) was defined as patients fulfilling the enrollment criteria, receiving at least 4 weeks of treatment or placebo, and having sufficient data to evaluate response. For those patients who did not complete 32 weeks of therapy, the last AQLQ score and other response parameters obtained on therapy were carried forward to 32 weeks. The per-protocol (PP) population was defined as patients fulfilling the enrollment criteria, receiving at least 32 weeks of treatment or placebo, and having a 32 -week evaluation. Additional evaluations of the adrenal axis were conducted in patients at the lead site only.

\section{Secondary Analyses}

Prespecified secondary analyses of AQLQ score comparison were as follows: the PP population and all randomized patients who took at least one dose of medication (ITT population); PP population with evidence of Aspergillus, Candida, Alternaria, Cladosporium, Penicillium, Trichophyton, and Botrytis sensitization; comparison of rhinitis scores; PP population by hospitalization in last 12 months or not; PP population without any evidence of alteration of glucocorticoid handling. Comparison of respiratory function testing, serum IgE, RAST titers, eosinophil counts, hospital visits and admissions for asthma, corticosteroid courses, relationship of itraconazole serum concentrations, and outcome and other exploratory analyses was also done.

\section{Data Handling}

All data were entered electronically into a bespoke database prepared by one of the authors (G.A.), using the MACRO database system (Infermed Ltd, London, UK). The data were held on a secure server used only for clinical data. Once each patient had completed the study, all the data were checked by the study nurses and data administrator before being locked. AQLQ scores were also manually checked before unblinding. Data were exported into the Access program (Microsoft, Redmond, WA) and then analyzed, using SPSS version 11.0, by the study statistician (J.Mo.), who unblinded the study. Subsequent sec- 


\begin{tabular}{|c|c|}
\hline \multirow{4}{*}{$\begin{array}{l}\text { - Severe asthma referrals } \\
\text { - Existing patient cohorts } \\
\text { - GP practice database search } \\
\text { - Direct patient referral from } \\
\text { media attention } \\
\text { - Skin test database } \\
\text { - RAST test database } \\
\text { - Research volunteer database } \\
\text { - Acute asthma admissions }\end{array}$} & $\begin{array}{l}\text { Patients notes/records } \\
\text { screened } \mathrm{N}=1847\end{array}$ \\
\hline & \begin{tabular}{l}
\multicolumn{1}{c}{$\downarrow$} \\
Patients actively \\
screened $\mathrm{N}=167$
\end{tabular} \\
\hline & $\downarrow$ \\
\hline & $\begin{array}{l}\text { Patients eligible } \\
N=73\end{array}$ \\
\hline
\end{tabular}

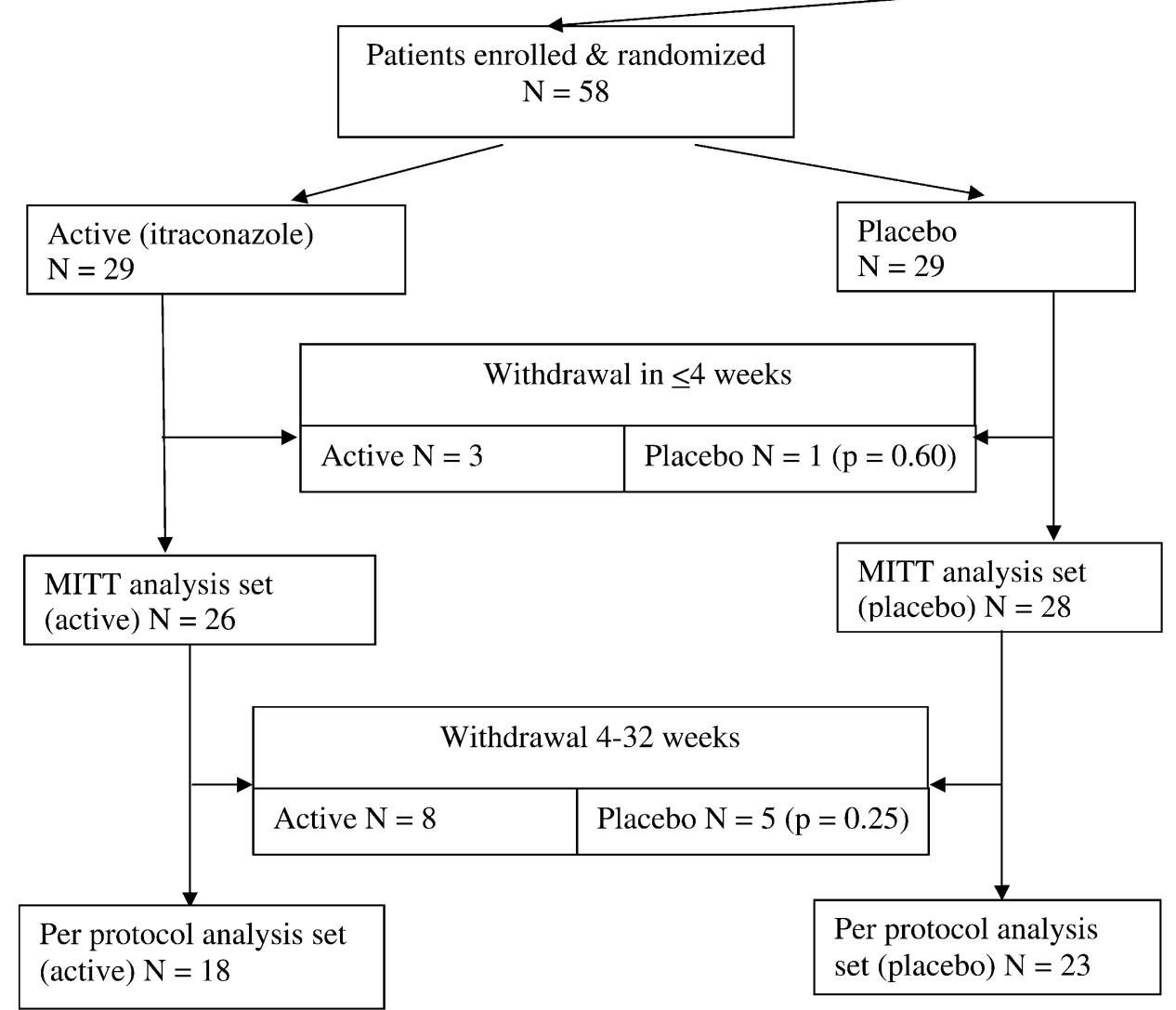

Figure 1. Study flowchart showing sources of patients, screening enrollment, and numbers of subjects discontinuing the study at various time points. ondary analyses were undertaken by G.A. and J.Mo. together, using an agreed-upon statistical analysis plan.

\section{RESULTS}

\section{Enrollment Characteristics}

The patients enrolled all had severe asthma, as shown in Table 1, with 41 of $58(71 \%)$ of patients receiving high-dose inhaled steroids, $6(10 \%)$ receiving continuous oral steroids, all but 1 receiving long-acting bronchodilators, and 24 (41\%) having been hospitalized in the previous 12 months for asthma and low mean AQLQ scores. Specifically, the median inhaled steroid dose and range (in beclomethasone equivalents) were similar between the groups: actively treated group: median, 1,600 $\mu \mathrm{g}$; range, 400-4,000; control group: median, 1,800 $\mu \mathrm{g}$; range, 400-4,000. The mean $\mathrm{FEV}_{1}$ was $71.5 \%$ of predicted, with a wide range of measurements. With respect to any fungal infection, $38(66 \%)$ recalled at least one superficial fungal infection, and rates of fungal sensitization were high. Rates of sensitization were highest to $A$. fumigatus and $C$. albicans as assessed by specific IgE (Table 1) or by skin prick test. Eosinophilia was seen in $19(33 \%)$ of patients and the geometric mean total $\mathrm{IgE}$ was $230 \mathrm{IU} / \mathrm{ml}$, with a wide individual variation.
No significant statistical differences or clinically significant differences were seen in any of the baseline characteristics shown in Table 1.

\section{Primary End Point}

Fifty-eight subjects were enrolled and 54 were assessed at Week 4 (MITT population). Another 13 subjects discontinued therapy before Week 32 and 41 patients completed 32 weeks of therapy (PP population) (see Figure 1). The AQLQ score improved significantly in both the MITT and PP populations. Those treated with antifungal therapy in the MITT population had a significantly higher increase in their AQLQ score of +0.82 (95\% confidence interval [CI]: 0.17, 1.46) (Figure 2A) when adjusted for baseline score; antifungal treatment subjects had a score of 4.92 compared with the placebo subjects, who had a score of 4.10 (analysis of covariance, $P=0.014$ ). In the PP population, the antifungal treatment group had a significantly higher increase in AQLQ score of +1.18 (95\% CI: 0.46, 1.89) (analysis of covariance, $P=0.002$ ); 5.36 compared with the placebo subjects 4.18 at 32 weeks, indicating better quality of life in antifungal-treated patients. Improvement in score in the $\mathrm{PP}$ population of at least 0.75 was seen in $61 \%$ of the antifungal 
TABLE 1. PATIENT DEMOGRAPHICS

\begin{tabular}{|c|c|c|}
\hline & \multicolumn{2}{|c|}{ Mean (range) or Percentage (no.) } \\
\hline & $\begin{array}{c}\text { Active } \\
(n=29)\end{array}$ & $\begin{array}{l}\text { Placebo } \\
(n=29)\end{array}$ \\
\hline Sex, male & $48 \%(14)$ & $48 \%(14)$ \\
\hline Age, yr & $49.2(18,79)$ & $51.7(19,76)$ \\
\hline \multicolumn{3}{|l|}{ Severity of asthma (BTS steps) ${ }^{\star \dagger}$} \\
\hline BTS step 5 & $3 \%(1)$ & $11 \%(3)$ \\
\hline BTS step 4 & $97 \%(28)$ & $89 \%(26)$ \\
\hline \multicolumn{3}{|l|}{ Superficial fungal infection (in past) } \\
\hline Oral thrush & $31 \%(9)$ & $41 \%(12)$ \\
\hline Genital thrush & $17 \%(5)$ & $21 \%(6)$ \\
\hline Fungal nail infection & $17 \%(5)$ & $21 \%(6)$ \\
\hline Fungal skin rash & $3 \%(1)$ & $3 \%(1)$ \\
\hline Athletes foot & $21 \%(6)$ & $31 \%(9)$ \\
\hline Any of preceding five & $62 \%(18)$ & $83 \%(20)$ \\
\hline \multicolumn{3}{|l|}{ Baseline positive fungal RAST/RAST or SPT } \\
\hline Aspergillus & $52 / 66 \%(15 / 19)$ & $52 / 79 \%(15 / 23)$ \\
\hline Cladosporium & $31 / 52 \%(9 / 15)$ & $17 / 28 \%(5 / 8)$ \\
\hline Alternaria* & $21 / 34 \%(6 / 10)$ & $32 / 45 \%(9 / 13)$ \\
\hline Penicillium & $39 / 48 \%(11 / 14)$ & $17 / 34 \%(5 / 10)$ \\
\hline Candida* & $52 / 66 \%(15 / 19)$ & $46 / 54 \%(13 / 15)$ \\
\hline Trichophyton $\$$ & $31 \%(8)$ & $19 \%(5)$ \\
\hline Botrytis* & $17 / 28 \%(5 / 8)$ & $14 / 32 \%(4 / 9)$ \\
\hline Baseline total serum IgE, IU/mI\| & $212(24,820)$ & $245(36,990)$ \\
\hline Baseline eosinophilia $\left(>0.4 \times 10^{9}\right)^{*}$ & $24 \%(7)$ & $43 \%(12)$ \\
\hline \multicolumn{3}{|l|}{ No. of hospitalizations in last $12 \mathrm{mo}$} \\
\hline Median (range) & $0(0,5)$ & $0(0,8)$ \\
\hline Percentage with none & $61 \%(14)$ & $83 \%(20)$ \\
\hline Requiring continuous oral steroids & $7 \%(2)$ & $14 \%(4)$ \\
\hline Requiring inhaled steroid at $>1,000 \mu \mathrm{g} / \mathrm{d}$ & $69 \%(20)$ & $72 \%(21)$ \\
\hline Median dose of inhaled steroid, $\mu \mathrm{g}$ beclomethasone equivalents (range) & $1,600(400-4,000)$ & $1,800(400-4,000)$ \\
\hline $\mathrm{FEV}_{1}, \mathrm{~L}^{*}$ & $2.15(0.5,4.35)$ & $2.14(0.65,3.85)$ \\
\hline $\mathrm{FEV}_{1}, \%$ predicted ${ }^{*}$ & $71.8(18,121)$ & $71.1(27,124)$ \\
\hline Peak expiratory flow A.M., L/min** & $322(120,795)$ & $312(101,558)$ \\
\hline Peak expiratory flow P.M., L/min ${ }^{\dagger \dagger}$ & $325(145,795)$ & $331(100,578)$ \\
\hline Baseline rhinitis score & $1.27(0,3.88)$ & $1.29(0,2.70)$ \\
\hline Baseline AQLQ score & $4.12(2.2,7.0)$ & $4.14(1,6.6)$ \\
\hline 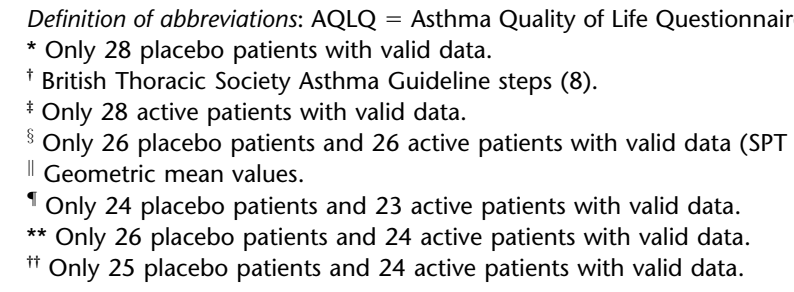 & $\Gamma \mathrm{S}=$ British Thoracic So & ; SPT = skin prick test. \\
\hline
\end{tabular}

treatment group, compared with $17 \%$ of the placebo group $(P=0.011)$.

\section{Secondary End Points}

Exacerbations were relatively infrequent, consistent with optimized antiasthma therapy before trial entry. Sixteen patients developed an asthma exacerbation or increased breathlessness during the study, 8 in each group, 3 in the first month on itraconazole and only 1 on placebo, with others throughout the 48 weeks of study. Four of these exacerbations resulted in hospital admission (see below). Eight patients developed a chest infection during the study, four in each group, all in Weeks 12-20.

There was also improvement in the rhinitis score among those taking antifungal therapy. At baseline the placebo subjects had a score of 1.25 and the antifungal group had a score of 1.27. More than $80 \%$ of patients reported some nasal symptoms at baseline, but the intensity was highly variable (as shown by the 95\% CIs; see Figure 2B) and changed considerably through the study. By the end of therapy at 32 weeks in the MITT population, the mean adjusted score for the placebo group had increased slightly to 1.42 , whereas the mean adjusted score of the antifungal group had decreased to $0.83(P=0.013$, analysis of covariance). The mean difference in score between the antifungal and placebo groups in the change between baseline and week 32 was 0.59 (95\% CI: $-1.06,-0.13$ ) (Figure 2B).

Total IgE fell at 32 weeks in the antifungal group, compared with the placebo group. The placebo group mean IgE value increased to $112 \%(95 \%$ CI: 95,131$)$ of baseline, compared with a fall to $73 \%(95 \%$ CI: 62, 86) in the antifungal group (Table 2). The antifungal group demonstrated a fall in geometric mean total $\mathrm{IgE}$ from 187 to $136 \mathrm{IU} / \mathrm{ml}$ and the placebo group a rise from 245 to $275 \mathrm{IU} / \mathrm{ml}$. These changes were apparent at 16 weeks. There was considerable variation in peripheral eosinophil counts, and no consistent trend.

There were modest changes in respiratory function as shown in Table 2. Morning PEF increased by a mean of $20.8 \mathrm{~L}$ in the antifungal group compared with a deterioration of $-5.5 \mathrm{~L}$ in the placebo group $(P=0.028)$, but neither evening PEF nor $\mathrm{FEV}_{1}$ improved significantly in either group.

When analyzed on the basis of the fungus to which patients were sensitized, all subgroups showed improvement, with some apparent variability per fungus, although some numbers are 

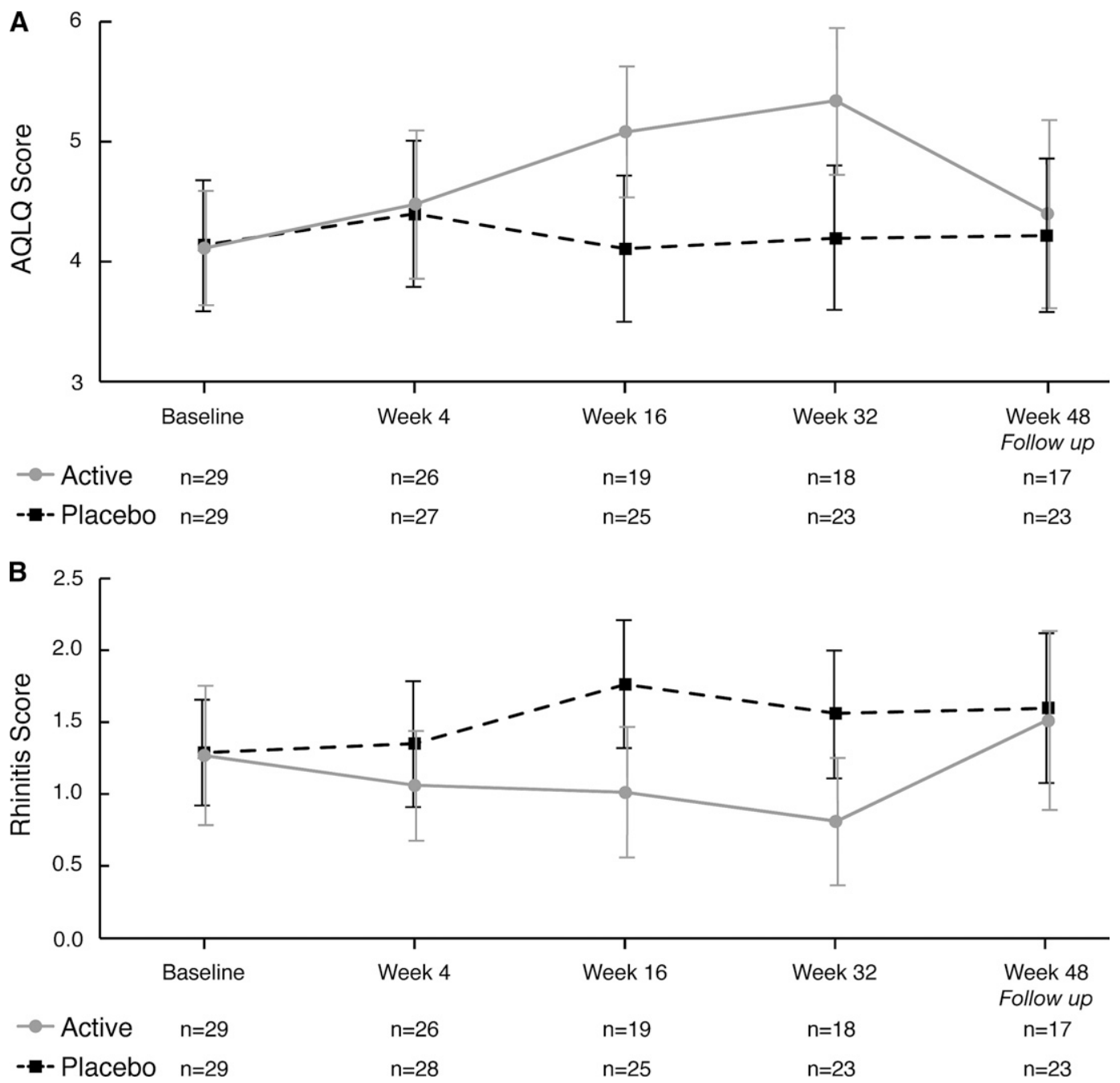

Figure 2. (A) Changes in Asthma Quality of Life Questionnaire (AQLQ) by time on study. Numbers of subjects studied are listed below each time point, and $95 \%$ confidence intervals are shown. Higher scores indicate improvement. The change at 32 weeks was +0.85 (95\% confidence interval $[95 \% \mathrm{Cl}]$ : $0.28,1.41$ ) in the antifungal therapy group and -0.01 (95\% Cl: $-0.43,0.42)$ in the placebo group. (B) Changes in rhinitis score by time on study. Numbers of subjects studied are listed below each time point (excluding those with zero scores at baseline), and $95 \%$ confidence intervals are shown. Higher scores indicate worsening condition. The change at 32 weeks was -0.43 (95\% Cl: $0.83,-0.02)$ in the antifungal therapy group and $0.17(95 \% \mathrm{Cl}$ : $-0.26,0.59)$ in the placebo group.

small. All analyses were done in the PP population and are shown in Table E1 in the online supplement.

At baseline, A. fumigatus was cultured from one placebo patient, Candida species from three other patients, and potentially pathogenic bacteria in another five patients. During the 48 -week study period, if a sputum sample was produced by a patient during the regular visits, it was sent for culture. During study, placebo patients produced 19 samples compared with 10 in the active treatment arm. Alternaria species was cultured from one itraconazole-treated patient and $A$. fumigatus from another treated with placebo, whereas Candida species was cultured from four placebo and two active treatment patients during the treatment phase. No patient was colonized with Pseudomonas species.

Four patients were admitted to hospital for asthma at least once during the study. Two of these patients were taking placebo, and

TABLE 2. RESPONSE TO THERAPY AT 32 WEEKS: MITT GROUP

\begin{tabular}{|c|c|c|c|}
\hline & \multicolumn{2}{|c|}{ Mean $(95 \% \mathrm{Cl})$ or Percentage $(n)$} & \multirow[b]{2}{*}{$P$ Value* } \\
\hline & Active & Placebo & \\
\hline Change in AQLQ score & $+0.85(0.28,1.41)$ & $-0.01(-0.43,0.42)$ & 0.014 \\
\hline Improvement in AQLQ score of $\geqslant 0.5$ & $54 \%(14)$ & $32 \%(9)$ & $0.18^{\dagger}$ \\
\hline Improvement in AQLQ score of $\geqslant 0.75$ & $54 \%(14)$ & $18 \%(5)$ & $0.013^{\dagger}$ \\
\hline Percentage change in total $\lg \mathrm{E}, \mathrm{IU} / \mathrm{m}^{\ddagger}$ & $-27 \%(-14 \%,-38 \%)$ & $+12 \%(-5 \%,+31 \%)$ & 0.001 \\
\hline Change in $\mathrm{FEV}_{1}, \mathrm{~L}$ & $-0.22(-0.56,0.11)$ & $-0.02(-0.16,0.11)$ & 0.22 \\
\hline Change in $\mathrm{FEV}_{1}, \%$ predicted & $-3.66(-9.39,2.08)$ & $0.13(-3.67,3.93)$ & 0.24 \\
\hline Change in average PEF (А.M.), $\mathrm{L} / \mathrm{min}$ & $20.8(3.5,38.1)$ & $-5.5(-21.6,10.7)$ & 0.028 \\
\hline Change in average PEF (P.M.), L/min & $16.8(1.5,35.2)$ & $8.9(-33.9,51.8)$ & 0.74 \\
\hline
\end{tabular}

Definition of abbreviations: AQLQ = Asthma Quality of Life Questionnaire; $\mathrm{Cl}=$ confidence interval; $\mathrm{MITT}=$ modified intent-totreat population; $\mathrm{PEF}=$ peak expiratory flow rate.

* Analysis of covariance.

${ }^{\dagger}$ Chi-square test.

${ }^{\ddagger}$ IgE results were skewed, and thus were log transformed before analysis to produce a reasonable approximation to a normal distribution. 
one was admitted once, the other nine times. Two patients were receiving antifungal therapy and each was admitted once; one had low serum itraconazole concentrations (mean, $1.25 \mathrm{mg} / \mathrm{L}$ ) and a much worse AQLQ score at 32 weeks, and the other discontinued early because of adverse events and was admitted to hospital after this.

Although it was hoped that maintenance inhaled and oral steroids would remain consistent through the study period, overall seven patients were documented as changing inhaled steroid dose through the study period: four increasing the inhaled steroid dose (three in the control group and one in the active treatment group) and three reducing the inhaled steroid dose (one in the control group and two in the active treatment group).

\section{Itraconazole Serum Concentrations}

Itraconazole plasma concentrations were determined in 58 patients at 2 weeks and in 41 patients at 20 weeks. Placebo subjects showed no detectable concentration. The 25 itraconazole subjects who received at least 4 weeks of treatment all had detectable levels, with individual means (over first $4 \mathrm{wk}$ ) ranging from 0.65 to $26.1 \mathrm{mg} / \mathrm{L}$ with a median of $8.9 \mathrm{mg} / \mathrm{L}$. There were no significant relationships between the mean itraconazole level per patient and the AQLQ score at Week 32 for the itraconazole group (Spearman correlation, $0.15 ; P=0.48$ ) or the change in AQLQ from Week 1 to Week 32 (Spearman correlation, -0.26; $P=0.22$ ) (see Figure E1 in the online supplement).

\section{Withdrawals and Adverse Events}

Seventeen patients withdrew from the study early: 11 in the active arm and 6 in the placebo arm, with 4 withdrawing in the first 4 weeks of study. Seven of these simply dropped out, moved away, or made a conscious decision to stop participating. Three patients cited ill health and seven reported significant adverse events as the reason for stopping. The adverse events included nausea $(n=2)$, edema $(n=2)$, breathlessness $(n=1)$, joint pains $(n=1)$, muscle weakness $(n=1)$, and cushingoid symptoms $(n=1)$, of which nausea and edema occurred in the placebo arm in one patient each. No severe adverse events occurred, apart from the hospitalizations for asthma, as described previously.

\section{Relapse}

Follow-up at 4 months after discontinuation of assigned therapy showed reversion of AQLQ score to nearly prestudy levels. In the MITT populations, placebo recipients had an overall change in quality of life at 12 months of +0.01 (95\% CI: $-0.34,0.37$ ) and those receiving antifungal therapy had a nonsignificant improvement of +0.19 (95\% CI: $-0.29,0.67)(P=0.54)$, compared with baseline. The same was true for the PP groups. Indeed, in the PP antifungal group, there was a reduction in AQLQ score 4 months after stopping antifungal therapy of -1.00 (95\% CI: $-1.67,-0.33)$, significantly different from placebo recipients $(P=0.01)$. In contrast, serum IgE changes during therapy were maintained at 4 months of follow-up compared with 32 weeks in the MITT population. The antifungal group had a marginal fall $(-0.1 \%)$ compared with the placebo group $(+4 \%)(P=0.7)$. Overall these data indicate that continuing antifungal therapy beyond 8 months is important for maintaining quality of life in these patients, and that $\mathrm{IgE}$ levels may not be useful to monitor for relapse.

\section{Corticosteroid Interaction Subgroup}

Twenty-seven patients were evaluated for adrenal axis perturbation by itraconazole. There was a nonsignificant trend in reduced plasma cortisol in the itraconazole-treated patients. Six of 13 itraconazole-treated patients had an interaction at 4 weeks (reduction in plasma cortisol to less than $50 \mathrm{nmol} / \mathrm{L}$ ) whereas in 7 patients there was no evidence of an interaction. No patients in the placebo group had a reduction in plasma cortisol to less than $50 \mathrm{nmol} / \mathrm{L}$. The mean AQLQ improvement at 32 weeks in the six patients with a likely itraconazole-corticosteroid interaction was $1.0(-0.8$ to +3.1$)$, compared with a mean improvement of $0.97(-1.2$ to +2.1$)$ in those without evidence of an interaction.

\section{DISCUSSION}

Although airborne fungal exposure is an everyday, lifelong occurrence, it is not intrinsically obvious that antifungal therapy would have any impact on asthma or rhinitis. In asthma and rhinitis, fungal sensitization is generally uncommon, except in those with severe asthma $(12,13)$, in contrast to frequent sensitization to house dust and feather mite, cat and dog allergens, and grass pollens. Clearly, antifungal therapy would have little or no impact on exposure to fungal allergens. However, one key distinguishing therapeutic characteristic of fungal exposure compared with other common allergenic exposures is that antifungal therapy exists.

The magnitude of the antifungal effect on symptoms of asthma seen in this study is promising. The AQLQ is a wellvalidated score of well-being, directly pertinent to those with asthma. In those who were able to continue treatment for 32 weeks ( $62 \%$ of subjects), the AQLQ rose by 1.18 , which compares with a rise of 0.15 with regular salbutamol (14), 0.55 with long-acting $\beta_{2}$-agonist therapy (14), and 0.42 with anti-IgE therapy $(15,16)$, the latter two heralded as major breakthroughs in the management of asthma. In accord with the improvements in pulmonary and nasal symptoms and patient well-being in our study, significant reductions in total serum $\operatorname{IgE}$ and a modest effect on morning PEF were also seen. The sample size for this study was small. Larger studies are required to assess more accurately the impact of antifungal therapy on lung physiology, and to confirm the results seen here. However, the impact of antifungal therapy in severe asthma is important as these patients have the most morbidity, lose more days of work, are more likely to be admitted to hospital and receive most corticosteroids with their attendant complications and are more likely to die of asthma than those with mild or moderate asthma.

Why is antifungal therapy effective for SAFS? Itraconazole is active against all the fungi to which the patients' sensitivity was tested. We know that in the uncommon disease ABPA, long-term colonization of the airways with A. fumigatus is demonstrable in most patients. A parallel situation in SAFS might explain why antifungal therapy is partially effective; colonization is reduced or eliminated. In SAFS, however, colonization is not easily demonstrable and many of the airborne fungi implicated are barely capable of growth at $37^{\circ} \mathrm{C}$ and inept at establishing themselves within the human host. One possibility is that there is sufficient colonization of the gastrointestinal tract and the upper or lower respiratory tract to provide antigenic stimulus to the immune system on a continuing basis. In support of this notion is the frequent finding of Candida species in human gut flora, which is increased after antibiotic therapy (17). In addition, the nose is a filter and may serve as a continual allergic reservoir of a wide diversity of culturable fungi (18). The lung in normal people is typically colonized by numerous fungi (19). Most airborne fungal structures are spores or conidia. These are robust, hydrophobic structures essentially devoid of allergenic proteins. Therefore fungal spores are unlikely 
to contribute to the pathogenesis of allergic asthma, with the possible exception of $C$. herbarum, as its hydrophobin protein (Cla H HCh-1) is allergenic (20). Another possibility is that shortterm repetitive exposure to viable hyphal fragments, which contain numerous diffusible allergens (21), is sufficient to drive allergic responses directly on an ongoing basis, and that exposure to fungal spores is irrelevant. A further possibility is that clinically apparent or subclinical infection with well-adapted cutaneous fungal pathogens such as Trichophyton species or gut-colonizing fungi such as Candida species directly impacts on asthma, and antifungal therapy reduces or eradicates such antigen exposure. In all cases, the supposition is that antifungal therapy reduces allergen exposure directly by killing viable filamentous fungi.

Another possibility is that itraconazole has a direct and profound immunologic effect. We have sufficient data to suggest a significant interaction between itraconazole and inhaled glucocorticoids, leading to excess corticosteroid exposure in half the patients evaluated. Interactions have been documented between itraconazole and inhaled steroids such as budesonide, and probably fluticasone $(22,23)$, but not prednisolone $(24,25)$. These interactions could damp allergic responses in these patients, but do not explain responses in those without an interaction, or why response was not observed in the first month of therapy, when the enhancement of corticosteroid exposure was seen. A direct major clinical effect on the immune system of the magnitude seen in this study has not been observed in the 20 years since itraconazole was first used in patients and is therefore unlikely, but conceivable. Unlike amphotericin B or the echinocandins, the azoles generally are relatively weak immunomodulators. Some direct immunologic effects of azoles have been reported in vitro, including suppression of type 2 cytokine production (IL-4 and IL-5) in T cells by itraconazole (26) and upregulation and/or production of numerous chemokines and inflammation-related cytokines, including IFN- $\gamma$ and tumor necrosis factor- $\alpha$, by voriconazole $(27,28)$. Itraconazole suppresses the elicitation phase of the allergic contact hypersensitivity reaction in hapten-challenged mice, thought to be by inhibition of IFN- $\gamma$ production from hapten-immunized effector T cells (29). Studies with itraconazole on polymorphonuclear leukocytes showed mixed results, but a strong inhibitory effect on the generation of allospecific cytolytic activity in human mixed lymphocyte culture is described (30). Fluconazole has even fewer immune effects and yet was effective for patients with asthma and Trichophyton sensitization (5), more consistent with a direct antifungal effect than an indirect immunologic effect. Given our response data in SAFS, the interaction of itraconazole with the immune system needs further experimental work but could contribute to the improvement observed.

The definition of SAFS used in this study was arbitrarily set to exclude all patients with ABPA, so that any criticism of simply repeating earlier therapeutic studies in ABPA was avoided. However, fungal sensitization and fungal allergy are imprecisely defined at present, and depend on manufacturerdependant reagents. We, and numerous others, have found a major disconnect between skin testing and RAST testing for fungi, in both qualitative and quantitative responses (31). This observation reflects the different strains of fungi used, the various preparative procedures, and the differing immune responses to different mixtures of allergens. We also excluded patients with positive $A$. fumigatus IgG antibodies (often described as precipitins) to reduce the possible overlap with ABPA. Many of these patients could benefit from antifungal therapy, even if they do not fulfill the criteria for ABPA, but we did not evaluate them. Given that $24 \%$ of those patients we actively screened for study inclusion manifested sensitization to only one fungus, and that there was a complete mismatch in skin test results and RAST results in $26 \%$ of patients, major improvements in diagnosing fungal allergy or sensitization are required. Indeed, it is possible that our current markers of fungal sensitization are so poor that many more patients with asthma would benefit from antifungal therapy than would be identified with our current definition of SAFS.

This trial was a randomized multicenter study conducted with a fixed budget. Recruitment fell below the initial target but despite this major improvements were seen in AQLQ, the primary outcome measure, largely because the magnitude of the benefit exceeded the pretrial power calculation response. However, the $95 \%$ confidence intervals for AQLQ and rhinitis score overlap at 32 weeks, which does not allow us to draw a definitive conclusion regarding the role of antifungal therapy in SAFS. The effect size seen on physiological parameters was modest at best (small improvement in morning peak flow only). Major reductions in $\mathrm{IgE}$, although not usually high at onset, were highly significant. Larger studies will be required to fully establish the place of antifungal therapy for severe asthma and more data on mechanism of action would assist in understanding the dichotomy between major symptomatic and $\mathrm{IgE}$ improvements, but few respiratory function changes. Possibly there are two populations within SAFS, a responder population and a nonresponder population, as the $54 \%$ of antifungal-treated patients who responded did so with large improvements in symptoms, whereas the other group of $46 \%$ were relatively unchanged or worse.

There was also a significant adverse event rate, with itraconazole implicated in some cases. Exacerbation rates were equivalent (and low) in both groups over the trial period. One patient was responsible for the large number of exacerbations in terms of hospitalizations for worsening of asthma, but this individual had experienced a similar number of exacerbations requiring hospitalization in the year before the study and hence she was continued in the study. Exacerbations were recorded at all routine study visits, and were defined by a need for a course of corticosteroids (or an increase in maintenance dose by more than $10 \mathrm{mg}$ of prednisolone). Individuals were supervised at one of four centers, but geographical origin was diverse and their primary and secondary care providers were not obligated to follow a specific protocol for exacerbations if they presented with acute symptoms in primary care or if they attended in the emergency department of their local hospital.

The primary aim of this proof-of-concept study was to contribute key data to the long-standing debate about whether there is any direct link between fungal exposure and asthma. Our data are most consistent with an important and poorly understood relationship. Our study results indicate that a new treatment approach using antifungal therapy in severe asthma is clinically useful, and that we have much to understand about the daily interaction between fungi and humans.

Conflict of Interest Statement: In the past 5 years, D.W.D has received grant support from Astellas, Merck, Pfizer, F2G, AstraZeneca, Indevus, and Basilea; he has been an advisor/consultant to Basilea, Vicuron (now Pfizer), Pfizer, Schering Plough, Indevus, F2G, Nektar, Daiichi, Sigma Tau, Astellas, Gilead, and York Pharma; he has been paid for talks on behalf of Schering, Astellas, Merck, and Pfizer; he holds founder shares in F2G, Ltd. and Myconostica, Ltd. B.R.O. does not have a financial relationship with a commercial entity that has an interest in the subject of this manuscript. G.P. does not have a financial relationship with a commercial entity that has an interest in the subject of this manuscript. F.C. does not have a financial relationship with a commercial entity that has an interest in the subject of this manuscript. Fees for attendance by G.T.A. at the 18th ECCMID were paid for by Pfizer. A.V. does not have a financial relationship with a commercial entity that has an interest in the subject of this manuscript. J.M. has received speaker fees in 2008 for meetings sponsored by Novartis $(£ 1,000)$, GlaxoSmithKline $(£ 500)$, and AstraZeneca $(£ 1,500)$. J.M. serves as a consultant to Myconostica Ltd. R.M.N. does not have a financial relationship with a commercial entity that has an interest in the subject of this manuscript. 
Acknowledgment: The authors are indebted to their colleagues who referred patients, in particular Gillian Miller in Congleton, Dr. McAndrew at Wrexham, Dr. Jenny Hoyle, and Dr. Rob Angus at Aintree and Newton Fold GP practice in Pendlebury, Manchester. The authors are also indebted to Susie Rushton (Pharmacy, Wythenshawe Hospital) for arranging and supervising blinded drug supplies; to the staff at the Regional Mycology Laboratory, Manchester for itraconazole assays and susceptibility tests; and to the staff of the Immunology Laboratory at St Mary's Hospital for IgE and RAST testing.

\section{References}

1. Denning DW, O'Driscoll BR, Hogaboam CM, Bowyer P, Niven RM. The link between fungi and severe asthma: a summary of the evidence. Eur Respir J 2006;27:615-626.

2. Denning DW, Van Wye J, Lewiston NJ, Stevens DA. Adjunctive therapy of allergic bronchopulmonary aspergillosis with itraconazole. Chest 1991;100:813-819.

3. Stevens DA, Schwartz HJ, Lee JY, Moskovitz BL, Jerome DC, Catanzaro A, Bamberger DM, Weinmann AJ, Tuazon CU, Judson $\mathrm{MA}$, et al. A randomized trial of itraconazole in allergic bronchopulmonary aspergillosis. $N$ Engl J Med 2000;342:756-762.

4. Wark PA, Hensley MJ, Saltos N, Boyle MJ, Toneguzzi RC, Epid GD, Simpson JL, McElduff P, Gibson PG. Anti-inflammatory effect of itraconazole in stable allergic bronchopulmonary aspergillosis: a randomized controlled trial. J Allergy Clin Immunol 2003;111:952-957.

5. Ward GW Jr, Woodfolk JA, Hayden ML, Jackson S, Platts-Mills TA. Treatment of late-onset asthma with fluconazole. J Allergy Clin Immunol 1999;104:541.

6. Riding DM, Denning D, Francis HC, Niven RM. The effect of itraconazole therapy in severe asthma patients allergic to Aspergillus: A retrospective audit [abstract 75]. Presented at the meeting Advances against Aspergillosis, September 9-11, 2004, San Francisco.

7. Denning DW, O'Driscoll BR, Powell G, Chew F, Atherton GT, Vyas A, Miles J, Morris J, Niven R. Proof of concept RCT of oral antifungal treatment for severe asthma with fungal sensitisation (SAFS). Abstract presented at the Interscience Conference on Antimicrobial Agents and Chemotherapy, September 17-20, 2007, Chicago.

8. British Thoracic Society, Scottish Intercollegiate Guidelines Network. British guidelines on the management of asthma. Thorax 2003;58: i1-i94.

9. Juniper EF, Guyatt GH, Epstein RS, Ferrie PJ, Jaeschke R, Hiller TK. Evaluation of impairment of health related quality of life in asthma: development of a questionnaire for use in clinical trials. Thorax 1992; 47:76-83.

10. Law D, Moore CB, Denning DW. A new bioassay for serum itraconazole concentrations using hydroxyitraconazole standards. Antimicrob Agents Chemother 1994;38:1561-1566.

11. Mucha SM, de Tineo M, Naclerio RM, Baroody FB. Comparison of montelukast and pseudoephedrine in the treatment of allergic rhinitis. Arch Otolaryngol Head Neck Surg 2006;132:164-172.

12. Zureik M, Neukirch C, Leynaert B, Liard R, Bousquet J, Neukirch F. Sensitisation to airborne moulds and severity of asthma: cross sectional study from European Community respiratory health survey. BMJ 2002;325:411-415.

13. O'Driscoll BR, Hopkinson LC, Denning DW. Mould sensitisation is common amongst patients with severe asthma requiring multiple hospital admissions in North West England. BMC Pulm Med 2005;5:4.

14. Juniper EF, Johnston PR, Borkhoff CM, Guyatt GH, Boulet LP, Haukioja A. Quality of life in asthma clinical trials: comparison of salmeterol and salbutamol. Am J Respir Crit Care Med 1995;151: 66-70.
15. Holgate S, Bousquet J, Wenzel S, Fox H, Liu J, Castellsague J. Efficacy of omalizumab, an anti-immunoglobulin $\mathrm{E}$ antibody, in patients with allergic asthma at high risk of serious asthma-related morbidity and mortality. Curr Med Res Opin 2001;17:233-240.

16. Buhl R, Solèr M, Matz J, Townley R, O’Brien J, Noga O, Champain K, Fox H, Thirlwell J, Della Cioppa G. Omalizumab provides long-term control in patients with moderate-to-severe allergic asthma. Eur Respir J 2002;20:73-78.

17. Odds FC. Candida and candidosis, 2nd ed. London: Baillière Tindall; 1988.

18. Ponikau JU, Sherris DA, Kern EB, Homburger HA, Frigas E, Gaffey TA, Roberts GD. The diagnosis and incidence of allergic fungal sinusitis. Mayo Clin Proc 1999;74:877-884.

19. Lass-Flörl C, Salzer GM, Schmid T, Rabl W, Ulmer H, Dierichi MP. Pulmonary Aspergillus colonization in humans and its impact on management of critically ill patients. Br J Haematol 1999;104:745-747.

20. Weichel M, Schmid-Grendelmeier P, Rhyner C, Achatz G, Blaser K, Crameri R. Immunoglobulin E-binding and skin test reactivity to hydrophobin HCh-1 from Cladosporium herbarum, the first allergenic cell wall component of fungi. Clin Exp Allergy 2003;33:72-77.

21. Green BJ, Tovey ER, Sercombe JK, Blachere FM, Beezhold DH, Schmechel D. Airborne fungal fragments and allergenicity. Med Mycol 2006;44(Suppl 1):S245-S255.

22. Raaska K, Niemi M, Neuvonen M, Neuvonen PJ, Kivisto KT. Plasma concentrations of inhaled budesonide and its effects on plasma cortisol are increased by the cytochrome P4503A4 inhibitor itraconazole. Clin Pharmacol Ther 2002;72:362-369.

23. Parmar JS, Howell T, Kelly J, Bilton D. Profound adrenal suppression secondary to treatment with low dose inhaled steroids and itraconazole in allergic bronchopulmonary aspergillosis in cystic fibrosis. Thorax 2002;57:749-750.

24. Varis T, Kivisto KT, Neuvonen PJ. The effect of itraconazole on the pharmacokinetics and pharmacodynamics of oral prednisolone. Eur J Clin Pharmacol 2000;56:57-60.

25. Lebrun-Vignes B, Archer VC, Diquet B, Levron JC, Chosidow O, Puech AJ, Warot D. Effect of itraconazole on the pharmacokinetics of prednisolone and methylprednisolone and cortisol secretion in healthy subjects. Br J Clin Pharmacol 2001;51:443-450.

26. Kanda N, Enomoto U, Watanabe S. Anti-mycotics suppress interleukin4 and interleukin-5 production in anti-CD3 plus anti-CD28-stimulated $\mathrm{T}$ cells from patients with atopic dermatitis. J Invest Dermatol 2001;117:1635-1646.

27. Simitsopoulou M, Roilides E, Likartsis C, Ioannidis J, Orfanou A, Paliogianni F, Walsh TJ. Expression of immunomodulatory genes in human monocytes induced by voriconazole in the presence of Aspergillus fumigatus. Antimicrob Agents Chemother 2007;51: 1048-1054.

28. Hohl TM, Van Epps HL, Rivera A, Morgan LA, Chen PL, Feldmesser M, Pamer EG. Aspergillus fumigatus triggers inflammatory responses by stage-specific $\beta$-glucan display. PLoS Pathog 2005;1:e30.

29. Ausaneya U, Kawada A, Aragane Y. Itraconazole suppresses an elicitation phase of a contact hypersensitivity reaction. $J$ Invest Dermatol 2006;126:1028-1035.

30. Pawelec G, Jaschonek K, Ehninger G. The anti-fungal agent itraconazole exerts immunosuppressive effects on alloreactivity but not on natural immunity in vitro. Int J Immunopharmacol 1991;13:875-879.

31. Smits WL, Letz KL, Evans TS, Giese JK. Evaluating the response of patients undergoing both allergy skin testing and in vitro allergy testing with the ImmunnoCAP technology system. J Am Acad Nurse Pract 2003; 15:415-423. 